\title{
Differences in risk factors for 3 types of stroke
}

\section{UK prospective study and meta-analyses}

\author{
Alison J. Price, DPhil,* F. Lucy Wright, DPhil,* Jane Green, DPhil, Angela Balkwill, MSc, Sau Wan Kan, MSc, \\ TienYu Owen Yang, DPhil, Sarah Floud, PhD, Mary E. Kroll, DPhil, Rachel Simpson, MB, BCh, \\ Cathie L.M. Sudlow, FRCP(E), Valerie Beral, FRS, and Gillian K. Reeves, PhD
}

Neurology ${ }^{\circledR}$ 2018;90:e298-e306. doi:10.1212/WNL.0000000000004856

\section{Abstract \\ Objective}

To compare associations of behavioral and related factors for incident subarachnoid hemorrhage and intracerebral hemorrhage and ischemic stroke.

\section{Methods}

A total of 712,433 Million Women Study participants without prior stroke, heart disease, or cancer reported behavioral and related factors at baseline (1999-2007) and were followed up by record linkage to national hospital admission and death databases. Cox regression yielded adjusted relative risks (RRs) by type of stroke. Heterogeneity was assessed with $\chi^{2}$ tests. When appropriate, meta-analyses were done of published prospective studies.

\section{Results}

After 12.9 (SD 2.6) years of follow-up, 8,128 women had an incident ischemic stroke, 2,032 had intracerebral hemorrhage, and 1,536 had subarachnoid hemorrhage. In women with diabetes mellitus, the risk of ischemic stroke was substantially increased (RR 2.01, 95\% confidence interval [CI] 1.84-2.20), risk of intracerebral hemorrhage was increased slightly (RR 1.31, 95\% CI 1.04-1.65), but risk of subarachnoid hemorrhage was reduced (RR 0.43, 95\% CI 0.26-0.69) (heterogeneity by stroke type, $p<0.0001$ ). Stroke incidence was greater in women who rated their health as poor/fair compared to those who rated their health as excellent/good (RR 1.36, 95\% CI 1.30-1.42). Among 565,850 women who rated their heath as excellent/good, current smokers were at an increased risk of all 3 stroke types, (although greater for subarachnoid hemorrhage [ $\geq 15$ cigarettes/d vs never smoker, RR 4.75, 95\% CI 4.12-5.47] than for intracerebral hemorrhage [RR 2.30, 95\% CI 1.94-2.72] or ischemic stroke [RR 2.50, 95\% CI 2.29-2.72]; heterogeneity $p<0.0001)$. Obesity was associated with an increased risk of ischemic stroke and a decreased risk of hemorrhagic stroke (heterogeneity $p<0.0001$ ). Metaanalyses confirmed the associations and the heterogeneity across the 3 types of stroke.

\section{Conclusion}

Classic risk factors for stroke have considerably different effects on the 3 main pathologic types of stroke.

\author{
Correspondence \\ Dr. Price \\ alison.price@lshtm.ac.uk
}




\section{Glossary}

$\mathbf{C I}=$ confidence interval; ICD-10 = International Classification of Diseases, 10 th revision; $\mathbf{N H S}=$ National Health Service; $\mathbf{R R}=$ relative risk.

Stroke is a leading cause of death and disability worldwide. ${ }^{1-3}$ Heart disease, hypertension, and diabetes mellitus, as well as behavioral and related factors such as smoking and obesity, are known to be associated with greater overall risk of stroke. ${ }^{4}$ The 3 main pathologic types of stroke are ischemic stroke, intracerebral hemorrhage, and subarachnoid hemorrhage, but evidence on the extent to which risk factors differ for these 3 different types is limited. Few studies have been sufficiently large to compare reliably the risk factors for the 3 types of stroke, and there is the potential problem of reverse causation bias whereby behavior changes caused by prior vascular disease or general poor health can distort associations between health-related behaviors and stroke.

We used data from a large prospective study of UK women to compare risk factors for ischemic stroke, intracerebral hemorrhage, and subarachnoid hemorrhage. To minimize biases associated with reverse causation, we excluded women with prior vascular disease or cancer from all analyses and, in some analyses, excluded women who rated their health as poor or fair and excluded the first 3 years of follow-up. To set our key findings in context, we reviewed the published evidence and, when appropriate, performed meta-analyses combining our results with those from previously published prospective studies.

\section{Methods}

\section{Study population}

Between 1996 and 2001, 1.3 million women joined the UK Million Women Study by completing a recruitment questionnaire on sociodemographic, health, and lifestyle characteristics. ${ }^{5}$ Study participants have been sent resurvey questionnaires every 3 to 5 years. Study questionnaires and details of the data and access policies can be viewed on the study website. ${ }^{6}$

\section{Standard protocol approvals, registrations, and patient consents}

The Multi-Centre Research Ethics Committee for Anglia and Oxford approved the study (REC reference 97/5/001). Participants provided written consent to follow-up through medical records.

\section{Exposures}

Baseline for these analyses was the 3-year resurvey questionnaire (completed on average 3.3 [SD 1.1] years after recruitment), which was the first time that participants were asked to rate their overall health (as excellent, good, fair, or poor), an important predictor of stroke risk. ${ }^{7}$ Women reported a history of diabetes mellitus and hypertension and whether they were being treated for it, heart disease, and other conditions, as well as current smoking, alcohol consumption, weight, and physical activity. Baseline information was used, except for region of residence, socioeconomic status, educational attainment, and height, which were recorded only at recruitment. Reported levels of physical activity were converted to excess metabolic equivalents. ${ }^{8}$ Because we could not distinguish between never and former drinkers, alcohol-associated risks were assessed in current drinkers at baseline.

\section{Outcomes}

Follow-up of all women in the cohort for deaths, emigration, and hospital admissions (inpatient and day case) was by electronic record linkage to routinely collected National Health Service (NHS) databases using each individual's NHS identification number and date of birth. Linked data were provided by NHS Digital (England) and NHS Information Services Division (Scotland). The data provided included date for each event and cause, coded to the ICD-10.

We defined first stroke as the earliest hospital admission with stroke, or death with stroke certified as the underlying cause, that occurred after baseline. Stroke outcome included any diagnosis of a specific stroke type: subarachnoid hemorrhage (ICD-10 code I60), intracerebral hemorrhage (ICD-10 code I61), and ischemic stroke (ICD-10 code I63). Women with $>1$ type of stroke at the first hospital admission $(\mathrm{n}=325)$ or with an unspecified type only (code I64, $\mathrm{n}=2,443$ ) were censored on that date.

\section{Statistical analysis}

Cox regression models were used to estimate hazard ratios, henceforth referred to as relative risks (RRs), and their 95\% confidence intervals (CIs), for incident stroke in relation to self-rated health, reported treatment for diabetes mellitus and hypertension, smoking status, alcohol consumption, body mass index, and physical activity. When $>2$ groups were compared, group-specific CIs were calculated using the variance of the log risk for each group. ${ }^{9}$

Women were excluded from the analysis in this order: if at baseline they did not report self-rated health $(n=25,682)$; if they had a hospital record of cerebrovascular disease 
$(n=4,027)$ or ischemic heart disease $(n=17,002)$; if they had reported a prior stroke or transient ischemic attack $(\mathrm{n}=$ $10,064)$ or prior heart disease $(n=37,628)$; if they had a prior cancer registration other than nonmelanoma skin cancer $(n=16,386)$; and if they had completed the baseline survey but were no longer registered with the NHS ( $\mathrm{n}=$ 105). After these exclusions, 712,433 women remained for analysis.

Person-years were calculated from the date the baseline questionnaire was completed to first hospital record of any stroke (ICD codes I60-I64), death, emigration, or March 31, 2015 (the latest date when complete information was available), whichever was earliest. Time in study was the underlying time variable, and analyses were stratified by year of birth (1930 or before, 1931 to 1949 in single years, 1950 or later) and of completion of the baseline questionnaire (2000 or before, 2001, 2002, 2003, 2004 or later) and adjusted for region of residence (10 geographical areas in the United Kingdom), socioeconomic status (5 levels of the Townsend index ${ }^{10}$ ), educational attainment (tertiary, secondary/technical, no formal qualifications ${ }^{11}$ ), and when appropriate for smoking (never, past, current <5, 5-9, $10-14,15-19,20-24, \geq 25$ cigarettes/d), alcohol consumption (none, $\leq 6,7-14, \geq 15$ drinks/wk), body mass in$\operatorname{dex}\left(<25,25-29.9, \geq 30 \mathrm{~kg} / \mathrm{m}^{2}\right)$, physical activity (thirds of excess metabolic equivalents), and menopausal hormone therapy (never, past, current). For adjustment variables, missing values were assigned to an unknown category and represented $<5 \%$ of the data in every variable. Sensitivity analyses excluding the first 3 years of follow-up were conducted.

We estimated log-linear trends in risk, when appropriate, scoring each category as the mean within-category measure. To allow for measurement error, regression dilution, and changes over time, estimates of trend in risk for tobacco and alcohol consumption, body mass index, and physical activity used mean remeasured values for the baseline categories being compared (appendix e-1, links.lww.com/WNL/A63). We used $\chi^{2}$ tests to assess heterogeneity across stroke types in the trend estimates. Statistical tests were 2 sided. Analyses used Stata version 14.0 (StataCorp, College Station, TX). ${ }^{12}$

\section{Systematic review and meta-analysis}

Recent reviews and meta-analyses have compared associations across the 3 stroke types with body mass index, ${ }^{13}$ hypertension, ${ }^{14,15}$ physical activity, ${ }^{16}$ and alcohol $^{17}$ and were not repeated here. We focused on diabetes mellitus and smoking, for which there had not been a recent relevant meta-analysis (appendix e-2, links.lww.com/WNL/A63, and figures e-1 and e-2, links.lww.com/WNL/A61, and tables e-1 and e-2, links.lww.com/WNL/A62, give search strategy and further details). Prospective studies were eligible for inclusion if they reported risk estimates for all of the 3 stroke types by diabetes mellitus or smoking in the same report, using the same analytic method for each type. Because the full consequences of the smoking epidemic in Asia have not yet emerged, ${ }^{18}$ studies were grouped into those from Asia and those from Europe or North America. We combined

Figure 1 Relative risk of stroke associated with diabetes mellitus and hypertension requiring treatment, reported at baseline

\section{Diabetes}

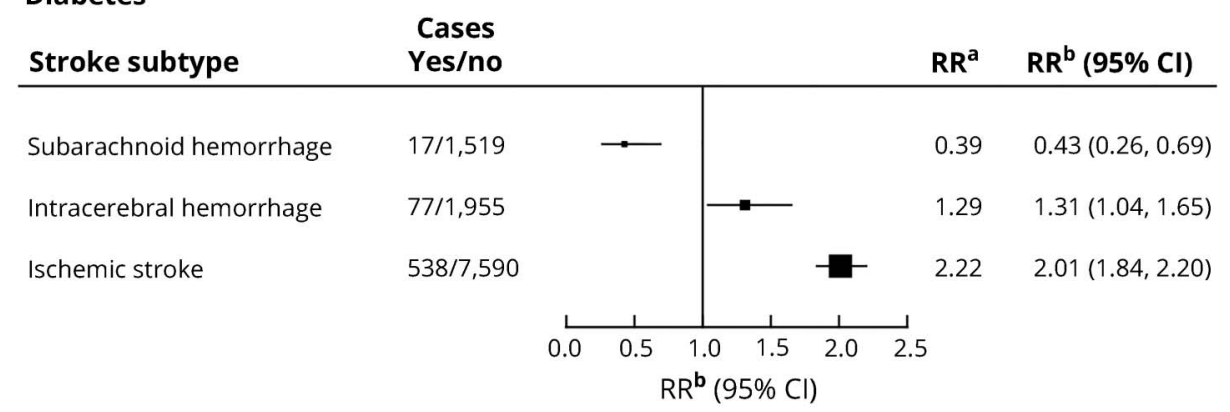

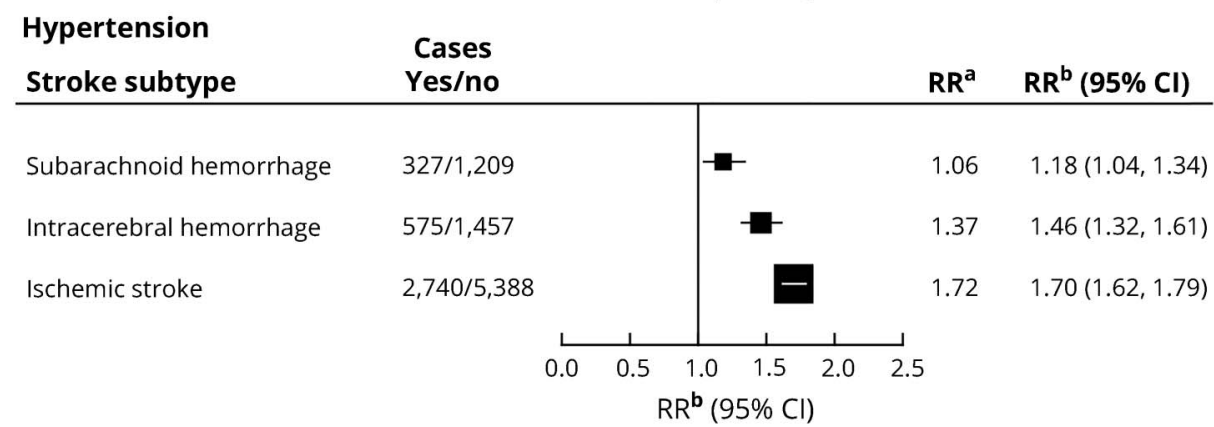

$\mathrm{RR}^{\mathrm{a}}$ is the relative risk for diabetes mellitus/hypertension compared to no diabetes mellitus/hypertension stratified by year of birth and calendar year at baseline and adjusted for region of residence, educational attainment, and socioeconomic status. $R R^{b}$ is the relative risk for diabetes mellitus/hypertension compared to no diabetes mellitus/hypertension, as for $\mathrm{RR}^{\mathrm{a}}$, and additionally adjusted for use of menopausal hormones, smoking, alcohol consumption, body mass index, and physical activity. $\mathrm{Cl}=$ confidence interval. 
type-specific stroke estimates across studies using inverse variance-weighted methods and tested heterogeneity across stroke types by $\chi^{2}$ tests.

\section{Results}

A total of 712,433 women without prior stroke, heart disease, or cancer and with information on self-rated health were followed up for 12.9 (SD 2.6) years on average. Their mean age at baseline was 59.8 (SD 4.9) years. There were large differences in behavioral and health characteristics in relation to self-rated health status (table e-3, links.lww.com/WNL/A62). Compared to women with excellent/good self-rated health, those with poor/fair self-rated health were more likely to be of lower socioeconomic status, obese, nondrinkers of alcohol, current smokers, and currently treated for diabetes mellitus or hypertension.

During follow-up, 11,696 women were admitted to hospital or died with a specified type of stroke: 8,128 had a first ischemic stroke, 2,032 had a first intracerebral hemorrhage, and 1,536 had a first subarachnoid hemorrhage. For only 4\% (460 of $11,696)$, the first record of a stroke was the underlying cause of death. Stroke was listed as the primary diagnosis in $>90 \%$ of the hospital admissions.
Among women reporting at baseline that they had diabetes mellitus that required treatment, compared to other women, the RR (adjusted for region of residence, deprivation, education, smoking, alcohol use, body mass index, physical activity and menopausal hormone therapy) was substantially increased for ischemic stroke (RR 2.01, 95\% CI 1.84-2.20), slightly but significantly increased for intracerebral hemorrhage (RR 1.31, 95\% CI 1.04-1.65), and substantially reduced for subarachnoid hemorrhage (RR 0.43, 95\% CI 0.26-0.69) (test for heterogeneity between the 3 stroke types $p<0.0001$ ). The exclusion of body mass index from the fully adjusted model for subarachnoid hemorrhage had a minimal effect (the diabetes mellitusassociated RR declined from 0.43 to 0.40 ). Among women who reported a history of hypertension requiring treatment, there was an increased risk of each stroke type: ischemic stroke (RR 1.70, 95\% CI 1.62-1.79), intracerebral hemorrhage (RR 1.46, 95\% CI 1.32-1.61), and subarachnoid hemorrhage (RR 1.18, 95\% CI $1.04-1.34)$, with significant heterogeneity by type $(p<0.0001$; figure 1).

In analyses adjusted for diabetes mellitus and hypertension at baseline and for region of residence, deprivation, education, smoking, alcohol use, body mass index, and physical activity, risk of stroke was significantly greater in women who rated their health as poor/fair than in those who rated their health

Figure 2 Relative risk of hemorrhagic and ischemic stroke associated with classic behavior-related vascular risk factors at baseline in those with excellent/good self-rated health

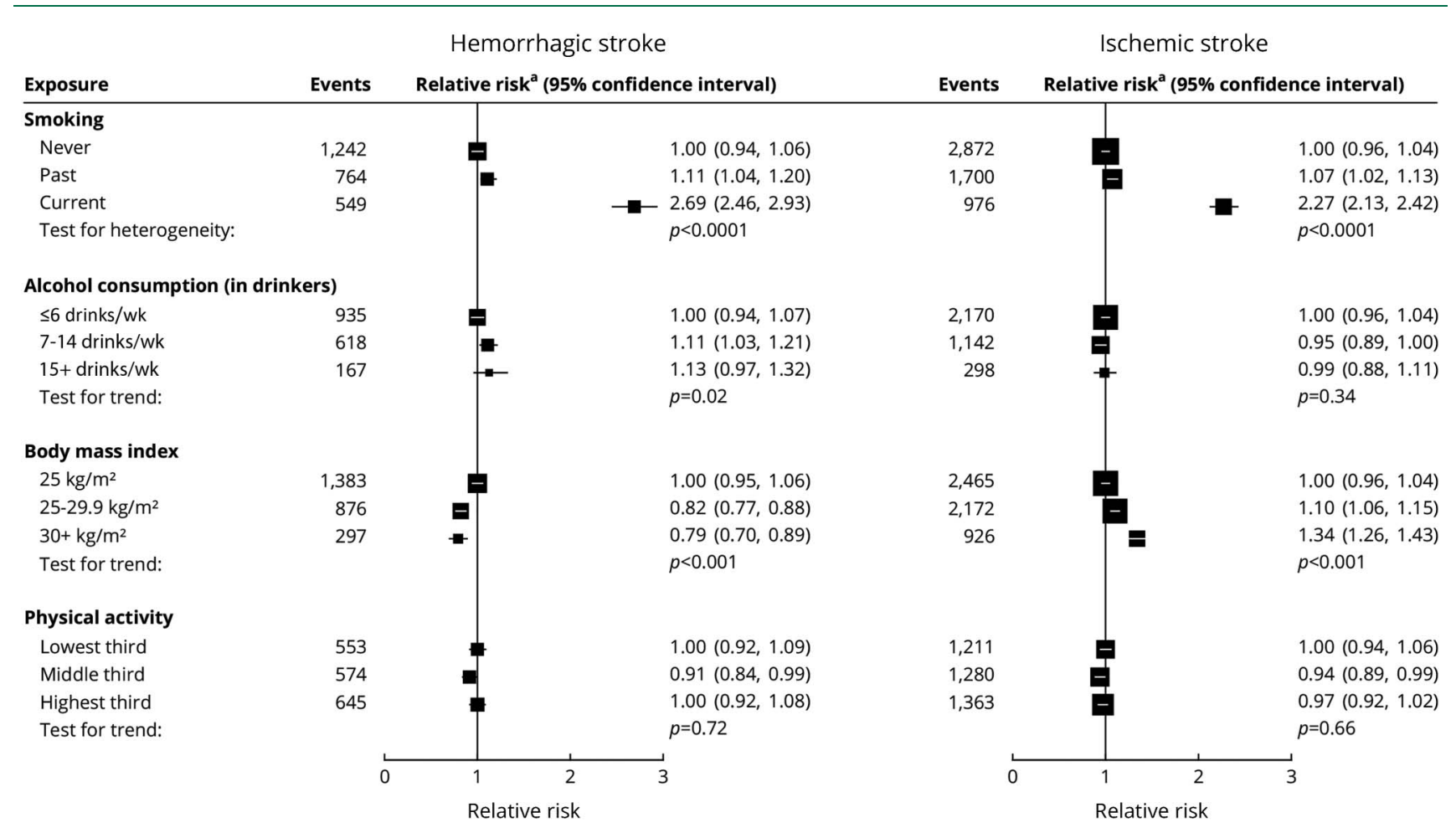

Relative risk ${ }^{a}$ is stratified by year of birth and calendar year at baseline and adjusted for region of residence, educational attainment, socioeconomic status, and use of menopausal hormones, and, when appropriate, for smoking, alcohol consumption, body mass index, and physical activity. Test for heterogeneity in the relative risk for current vs never smoking between hemorrhagic and ischemic stroke: $p=0.01$. Test for heterogeneity in trend between hemorrhagic and ischemic stroke: alcohol consumption $p=0.02$, body mass index $p<0.0001$, and physical activity $p=0.59$. 
as excellent/good (RR 1.36, 95\% CI 1.30-1.42). To minimize the potential for reverse causation bias resulting from changes in behavior due to preexisting ill health, analyses of associations of behavioral and related factors with risk of stroke were restricted to the 565,850 women who rated their health at baseline as excellent or good.

In women with excellent/good self-rated health, current smoking was associated with an increased risk of both hemorrhagic and ischemic stroke (figure 2), with risk greater for subarachnoid than for intracerebral hemorrhage (figure 3 ). The associations are dose dependent (figure 4), with RRs for $\geq 15$ cigarettes/d being 4.75 (95\% CI 4.12-5.47) for subarachnoid hemorrhage, 2.30 (95\% CI 1.94-2.72) for intracerebral hemorrhage, and 2.50 (95\% CI 2.29-2.72) for ischemic stroke (heterogeneity across stroke types $p<0.0001$; figure 4).

Adiposity was associated with an increased risk of ischemic stroke but reduced risk of hemorrhagic stroke, with no significant difference in the associations between subarachnoid hemorrhage and intracerebral hemorrhage.

Alcohol consumption in drinkers showed little association with either ischemic or hemorrhagic stroke, although there was some suggestion for increased risk of intracerebral hemorrhage with higher alcohol consumption. There was no significant association between physical activity and risk for any stroke type.

In analyses of women reporting good/excellent self-rated health, exclusion of the first 3 years of follow-up had little effect on the findings for any of the risk factors considered (figure e-3, links.lww.com/WNL/A61).

Results corresponding to figures 2 and 3 for women rating their health as poor/fair at baseline are shown in figures e-4 and e-5, links.lww.com/WNL/A61. In women with poor/ fair health, certain associations differed from those in women with excellent/good health in ways that were consistent with poor health having changed behavior. In particular, associations with smoking were considerably attenuated, and associations with physical activity were qualitatively different.

\section{Systematic review and meta-analysis}

For diabetes mellitus, we identified 2 eligible studies ${ }^{18,19}$ (figure 5A). Combined results from 3 studies (including ours) showed about a halving in the diabetes mellitus-associated RR of

Figure 3 Relative risk of subarachnoid and intracerebral hemorrhage associated with classic behavior-related factors at baseline in those with excellent/good self-rated health

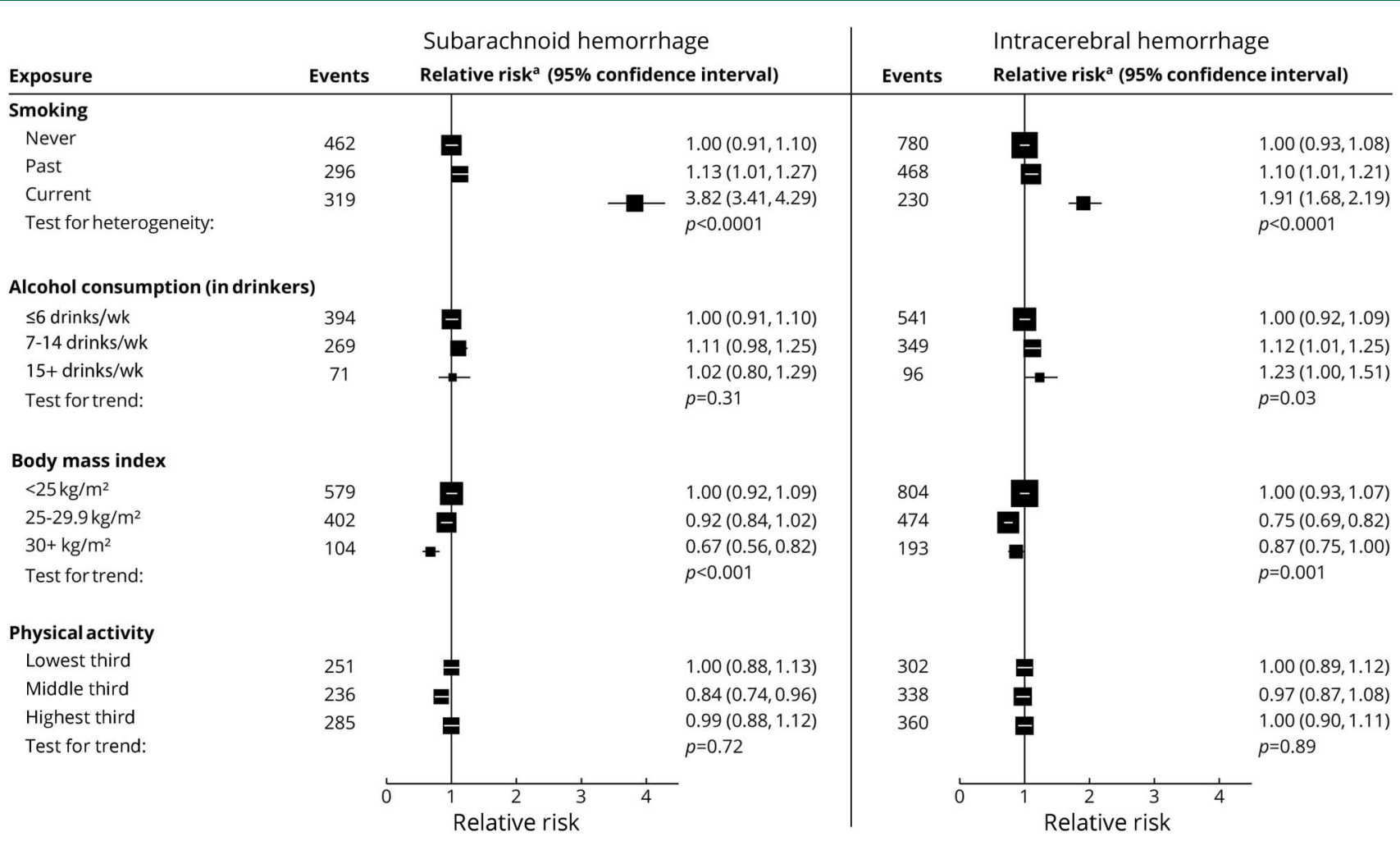

Relative risk ${ }^{a}$ is stratified by year of birth and calendar year at baseline and adjusted for region of residence, educational attainment, socioeconomic status, and use of menopausal hormones, and, when appropriate, for smoking, alcohol consumption, body mass index, and physical activity. Test for heterogeneity in the relative risk for current vs never smoking between subarachnoid hemorrhage and intracerebral hemorrhage: $p<0.0001$. Test for heterogeneity in trend between subarachnoid hemorrhage and intracerebral hemorrhage: alcohol consumption $p=0.51$, body mass index $p=0.54$, and physical activity $p=0.86$. 
Figure 4 Relative risk of stroke type by amount smoked in current smokers at baseline in those with excellent/good self-rated health

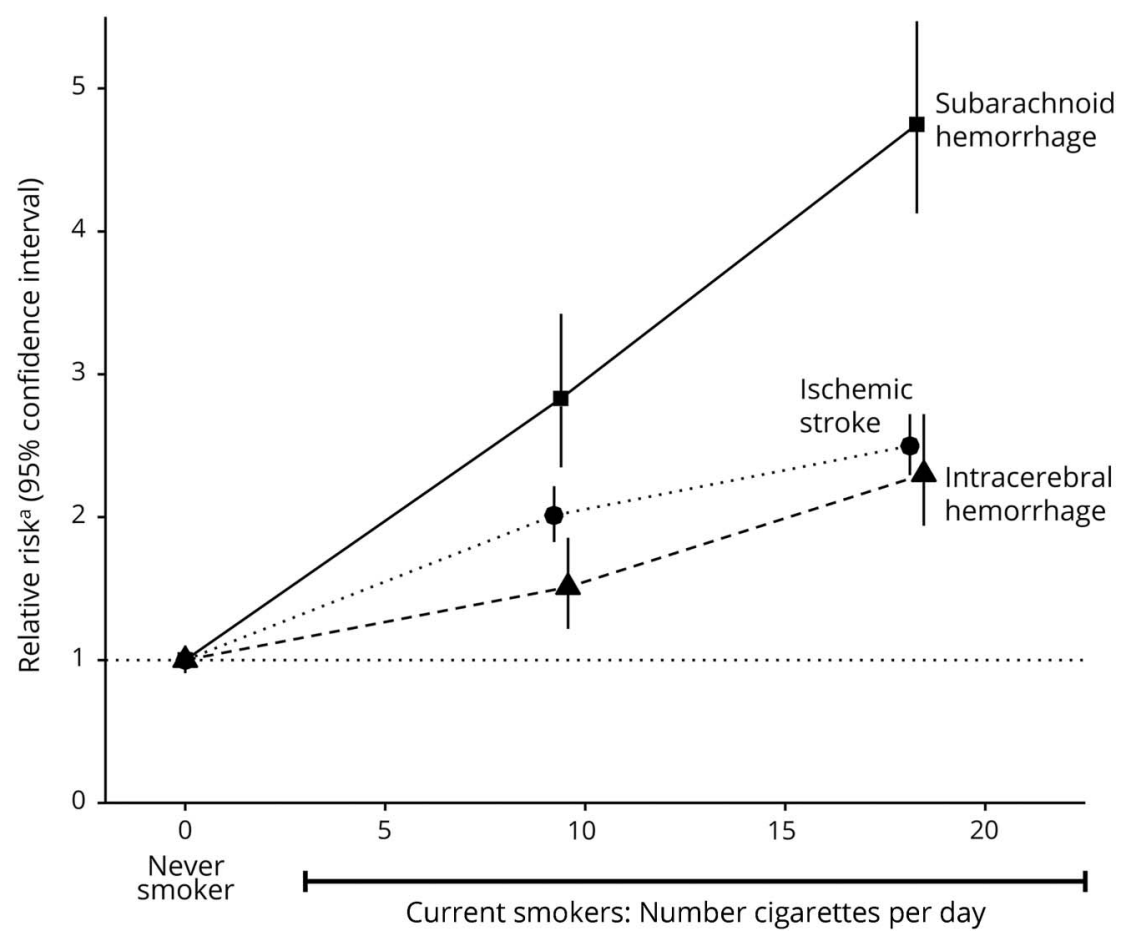

Relative risk ${ }^{a}$ is the relative risk in current vs never smokers stratified by year of birth and calendar year at baseline and adjusted for region of residence, educational attainment, socioeconomic status, use of menopausal hormones, alcohol consumption, body mass index, and physical activity. subarachnoid hemorrhage (RR 0.48, 95\% CI 0.34-0.69), a modest increase in risk of intracerebral hemorrhage (RR 1.25, 95\% CI 1.06-1.46), and almost a doubling in risk of ischemic stroke (RR 1.95, 95\% CI 1.82-2.10; test for heterogeneity across the 3 types $p<0.001)$.

For current smoking, 5 previously published studies were identified, 3 from Asia ${ }^{19-21}$ and 2 from Europe or North America. $^{22,23}$ Combined RRs from the 6 studies (including ours) were increased for all stroke types (figure 5B). The RRs associated with current smoking were consistently higher in Europe/North America than in Asia, but in both regions, current smokers were at greater risk for subarachnoid hemorrhage (RR 3.46 and 2.01 for Europe/North America and for Asia, respectively) than for intracerebral hemorrhage ( $R R$ 1.77 and 1.09, respectively) or for ischemic stroke (RR 2.17 and 1.58 , respectively; test for heterogeneity across the 3 types $p<0.001$ for each region).

\section{Discussion}

In this large prospective epidemiologic study with almost 12,000 incident strokes of the 3 main pathologic types, we found considerable heterogeneity in the risks of subarachnoid hemorrhage, intracerebral hemorrhage, and ischemic stroke associated with a history of diabetes mellitus, a history of hypertension, current smoking, and adiposity. Physical activity and alcohol consumption were not strongly related to risk of any of the 3 types of stroke.
A meta-analysis of results from this and previously published prospective studies showed about a halving in the risk of subarachnoid hemorrhage associated with a history of diabetes mellitus. In contrast, there was a small increase in risk of intracerebral hemorrhage and a doubling in risk of ischemic stroke. The increased risk of ischemic stroke in people with diabetes mellitus is well established and believed to have a mechanism similar to that for ischemic heart disease. $^{24} \mathrm{~A}$ reduced risk of subarachnoid hemorrhage associated with diabetes mellitus has been reported in several retrospective and prospective studies, ${ }^{25}$ but few prospective studies have directly compared diabetes mellitus-associated risk for subarachnoid hemorrhage with the risk for the other types of stroke. ${ }^{26,27}$ The mechanism for the observed reduction in diabetes mellitus-associated risk for subarachnoid hemorrhage is not clear.

In the interpretation of our findings on risk of stroke in relation to hypertension, it is important to note that our analyses were necessarily based on whether a woman reported having hypertension that required treatment at baseline because information on blood pressure was not generally available. Most previous studies that have examined the relationship between hypertension and stroke risk have used measured blood pressure as the exposure, ${ }^{13,14,27}$ often reporting stronger relationships for hemorrhagic than ischemic stroke. ${ }^{15,28}$ The extent to which our findings for a history of hypertension are relevant to the corresponding pattern in relation to measured blood pressure is uncertain. 
Figure 5 Meta-analysis of prospective studies that reported the risk of the 3 types of stroke

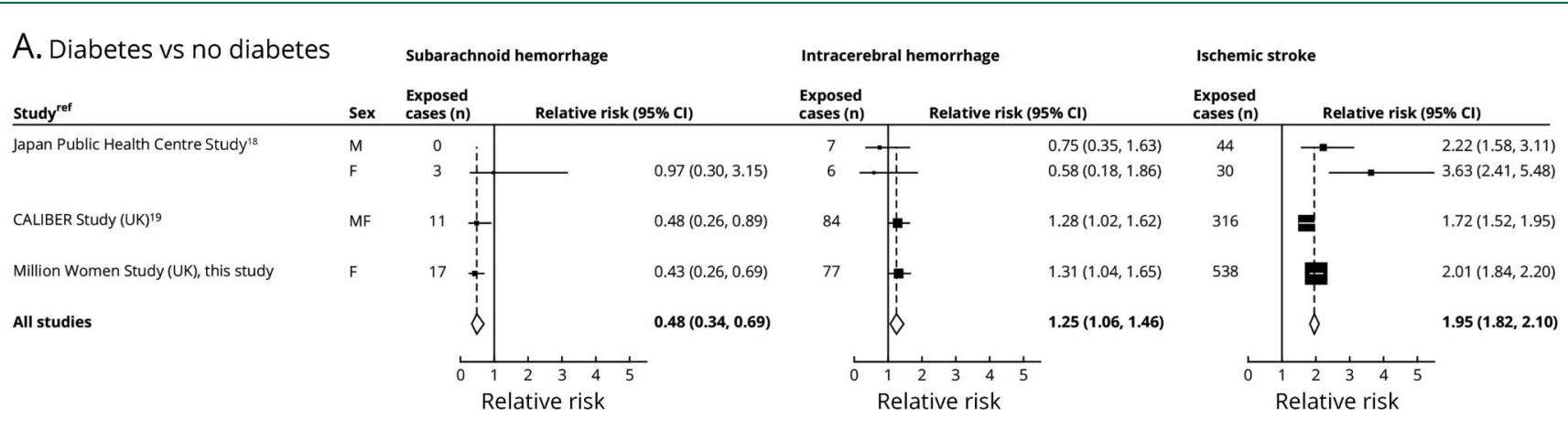

B. Current vs never smokers

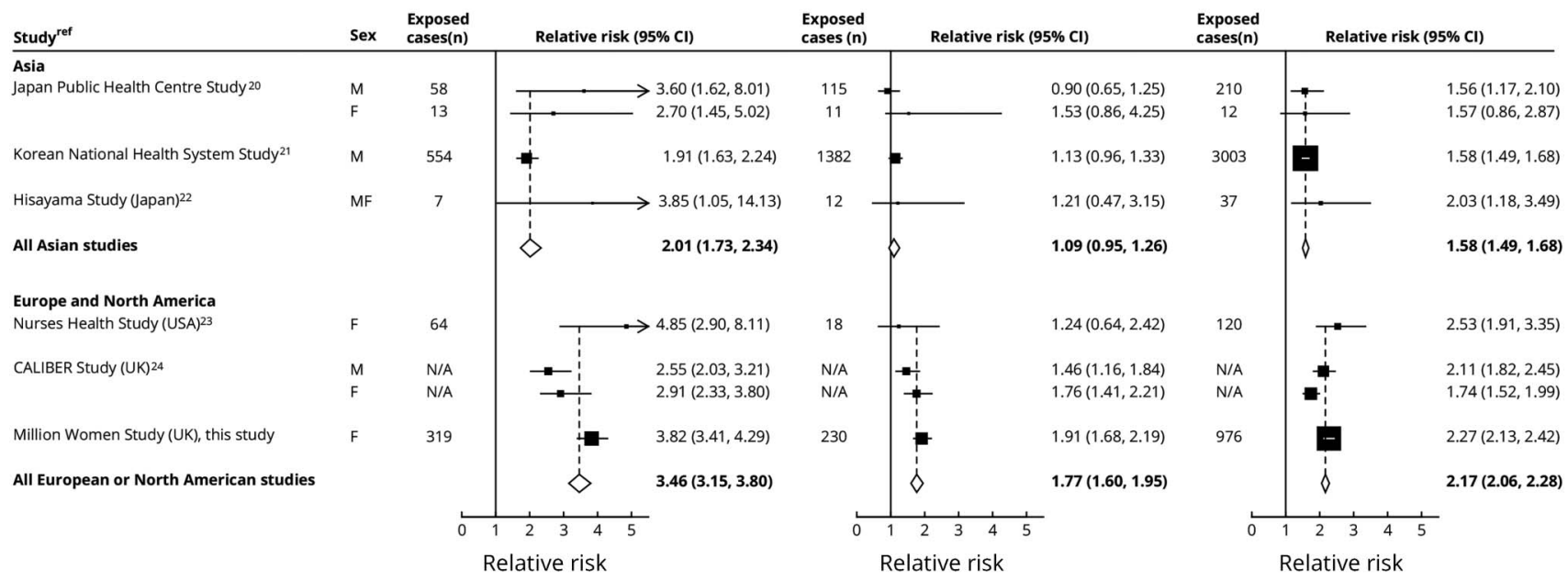

(A) Diabetes mellitus vs no diabetes mellitus (heterogeneity between stroke types $p<0.001$ ) and (B) current vs never smokers (heterogeneity between stroke types $p<0.001$ in Asian studies and $p<0.001$ in European and North American studies). $\mathrm{Cl}=$ confidence interval; $\mathrm{RR}=$ relative risk.

The risk of each of the 3 stroke types was increased in current smokers, and the relationships were dose dependent. In every study in our meta-analysis, the magnitude of the smokingassociated risk was greater for subarachnoid hemorrhage than for intracerebral hemorrhage or ischemic stroke. These results are consistent with those from meta-analyses restricted to subarachnoid hemorrhage alone. ${ }^{25,29}$ The distribution of stroke types is known to differ between populations of Asian and European origin, ${ }^{30}$ with hemorrhagic strokes making up a greater proportion of all strokes in Asia. The differences in smoking-related risks seen here between studies from Europe and North America and those from Asia may reflect the fact that the full health consequences of the smoking epidemic in Asia have not yet emerged. ${ }^{18}$ Likewise, any apparent differences by sex in smoking-related risks of stroke reported in the past may reflect the fact that the full health consequences of the smoking epidemic emerged later in women than men; in the 21st century, the effects of smoking are similar in men and women. $^{31}$

For obesity, we previously reported an increased risk of ischemic stroke, and decreased risk of both types of hemorrhagic stroke, which is confirmed with updated data here, consistent with the meta-analysis of results from published prospective studies. $^{13}$

Some have reported a reduced risk of ischemic and hemorrhagic stroke associated with moderate to vigorous physical activity $^{8,16}$ but did not take previous ill health into account. Any apparent increase in stroke risk associated with inactivity may be due to reverse causation, with women reducing their activity because of poor health, which itself is associated with an increased risk of stroke. Our finding of an apparently lower risk of stroke with greater physical activity only in women rating their health as poor or fair at baseline supports this view.

Levels of alcohol consumption in our participants are relatively low, and we found limited evidence for an association between amount consumed and stroke risk, although a modestly increased risk was observed for intracerebral hemorrhage with higher alcohol consumption. We did not include nondrinkers in our trend tests because we cannot distinguish between never drinkers and former drinkers. Drinking no alcohol was reported much more commonly in those with poor/fair health than in those with excellent/good health because some may have reduced their consumption because of ill health. A recent review 
and meta-analysis ${ }^{17}$ found modestly increased risks for each stroke type in heavy drinkers.

Strengths of the Million Women Study include its prospective design, large size, and virtually complete long-term follow-up by record linkage to routinely collected NHS databases. Only $\approx 1 \%$ of participants were lost to follow-up, largely because of emigration, but they were included in the analyses up to the date when they were lost. We found full agreement with primary care records for $96 \%$ of hospital admissions for subarachnoid hemorrhage, $78 \%$ for intracerebral hemorrhage, and $86 \%$ for cerebral ischaemia. ${ }^{32}$

By excluding women with prior stroke, ischemic heart disease, or cancer, and those with self-rated poor/fair health at baseline, we minimized the potential bias associated with reverse causation for health-related behavioral factors. To account for measurement error, regression dilution, and changes over time, we used remeasured values of these factors in the estimation of trends in risk.

Our analyses were confined to strokes that resulted in hospital admission. Because hemorrhagic strokes are more likely than ischemic strokes to result in a hospital admission probably because of their relative severity, hemorrhagic stroke may well be overrepresented here compared with the general population. This should not, however, bias findings for stroke types. When we compared primary care and hospital admission data for a subset of the cohort, only 6 of 864 participants without a hospital admission for stroke had any record of a cerebrovascular event in their primary care records. ${ }^{32}$

Although we adjusted for age, socioeconomic status, and lifestyle factors, we cannot rule out residual confounding by unmeasured factors. The measures available to us for hypertension were limited and make it difficult to interpret our findings. We studied UK women only, but our meta-analyses, which found similar results, included men and populations from other regions of the world.

Taken together, results from this large cohort of UK women and from meta-analyses show substantial heterogeneity in the associations of diabetes mellitus, smoking, and adiposity with incident subarachnoid hemorrhage, intracerebral hemorrhage, and ischemic stroke. These findings confirm that the 3 main pathologic types of stroke have considerably different etiologies.

\section{Author contributions}

Alison Price and Lucy Wright conducted the literature review, drafted the manuscript, advised on analysis and interpretation of the data. Angela Balkwill and Sau Wan Kan analyzed the data. Jane Green conceived and designed the study, contributed to the analysis, interpretation of the data, and revision of the manuscript. Owen Yang prepared the meta-analysis figures and revised the manuscript. Sarah Floud, Mary Kroll, and Rachel Simpson contributed to the analysis, interpretation of the data, and revision of the manuscript. Cathie Sudlow advised on analysis, interpretation of the data, and revision of the manuscript. Valerie Beral conceived and designed the study, contributed to the analysis, interpretation of the data, and revision of the manuscript. Gillian Reeves conceived and designed the study, contributed to the analysis, interpretation of the data and revision of the manuscript. She is the study guarantor.

\section{Acknowledgment}

The authors thank the women recruited to the Million Women Study, the staff of collaborating NHS Breast Screening Centres and General Practices, Benjamin Cairns (statistical advice), and Adrian Goodill (generating figures and editorial input). They also thank NHS Digital in England (www.digital.nhs.uk) and the Information Services Division in Scotland (www.isdscotland.org) for linked health data.

\section{Study funding}

This study was funded by the UK Medical Research Council and Cancer Research UK. Alison Price is a Wellcome Trust Research Fellow.

\section{Disclosure}

The authors report no disclosures relevant to the manuscript. Go to Neurology.org/N for full disclosures.

Received May 4, 2017. Accepted in final form October 10, 2017.

\section{References}

1. Krishnamurthi RV, Feigin VL, Forouzanfar $\mathrm{MH}$, et al. Global and regional burden of first-ever ischaemic and haemorrhagic stroke during 1990-2010: findings from the Global Burden of Disease Study 2010. Lancet Glob Health 2013;1:e259-e281.

2. Bennett DA, Krishnamurthi RV, Barker-Collo $S$, et al. The global burden of ischemic stroke: findings of the GBD 2010 study. Glob Heart 2014;9:107-112.

3. Krishnamurthi RV, Moran AE, Forouzanfar MH, et al. The global burden of hemorrhagic stroke: a summary of findings from the GBD 2010 study. Glob Heart 2014;9: 101-106.

4. Meschia JF, Bushnell C, Boden-Albala B, et al. Guidelines for the primary prevention of stroke: a statement for healthcare professionals from the American Heart Association/American Stroke Association. Stroke 2014;45:3754-3832.

5. Reeves GK, Pirie K, Beral V, Green J, Spencer E, Bull D. Cancer incidence and mortality in relation to body mass index in the Million Women Study: cohort study. BMJ 2007;335:1134-1139.

6. The Million Women Study. Available at: http://www.millionwomenstudy.org/ questionnaires/. Accessed January 15, 2016.

7. Howard G, McClure LA, Moy CS, et al. Self-reported stroke risk stratification. Stroke 2017;48:1737-1743

8. Armstrong MEG, Green J, Reeves GK, Beral V, Cairns BJ. Frequent physical activity may not reduce vascular disease risk as much as moderate activity: large prospective study of women in the United Kingdom. Circulation 2015;131:721-729.

9. Plummer M. Improved estimates of floating absolute risk. Stat Med 2004;23:93-104.

10. Townsend P, Phillimore P, Beattie A. Health and Deprivation: Inequality and the North. London: Croom Helm; 1988

11. Floud S, Balkwill A, Moser K, et al. The role of health-related behavioural factors in accounting for inequalities in coronary heart disease risk by education and areadeprivation: prospective study of 1.2 million UK women. BMC Med 2016;14:145.

12. StataCorp. Statistical Software, Release 14.0. College Station: StataCorp; 2015.

13. Kroll ME, Green J, Beral V, et al. Adiposity and ischemic and hemorrhagic stroke. Neurology 2016;87:1473-1481.

14. Lewington S, Clarke R, Qizilbash N, Peto R, Collins R. Age-specific relevance of usual blood pressure to vascular mortality: a meta-analysis of individual data for one million adults in 61 prospective studies. Lancet 2002;360:1903-1913.

15. Blood pressure and cardiovascular disease in the Asia Pacific region. J Hypertens 2003; $21 \cdot 707-716$.

16. Wendel-Vos GCW, Schuit AJ, Feskens EJM, et al. Physical activity and stroke: a metaanalysis of observational data. Int J Epidemiol 2004;33:787-798.

17. Larsson SC, Wallin A, Wolk A, Markus HS. Differing association of alcohol consumption with different stroke types: a systematic review and meta-analysis. BMC Med 2016;14:178 
18. Chen X, Zhou L, Zhang Y, et al. Risk factors of stroke in Western and Asian countries: a systematic review and meta-analysis of prospective cohort studies. BMC Public Health 2014;14:776.

19. Mannami T, Iso H, Baba S, et al. Cigarette smoking and risk of stroke and its subtypes among middle-aged Japanese men and women: the JPHC study cohort I. Stroke 2004; $35: 1248-1253$.

20. Lawlor DA, Song YM, Sung J, Ebrahim S, Smith GD. The association of smoking and cardiovascular disease in a population with low cholesterol levels: a study of 648,346 men from the Korean National Health System Prospective Cohort Study. Stroke 2008:39:760-767.

21. Hata J, Doi Y, Ninomiya T, et al. Combined effects of smoking and hypercholesterolemia on the risk of stroke and coronary heart disease in Japanese: the Hisayama study. Cerebrovasc Dis 2011;31:477-484.

22. Kawachi I, Colditz GA, Stampfer MJ, et al. Smoking cessation and decreased risk of stroke in women. JAMA 1993;269:232-236.

23. Pujades-Rodriguez $\mathrm{M}$, George J, Shah $\mathrm{AD}$, et al. Heterogeneous associations between smoking and a wide range of initial presentations of cardiovascular disease in 1937360 people in England: lifetime risks and implications for risk prediction. Int J Epidemiol 2015;44:129-141.

24. Grundy SM, Pasternak R, Greenland P, Smith S Jr, Fuster V. Assessment of cardiovascular risk by use of multiple-risk-factor assessment equations: a statement for healthcare professionals from the American Heart Association and the American College of Cardiology. Circulation 1999;100:1481-1492.
25. Feigin VL, Rinkel GJE, Lawes CMM, et al. Risk factors for subarachnoid hemorrhage: an updated systematic review of epidemiological studies. Stroke 2005; 36:2773-2780.

26. Cui R, Iso H, Yamagishi $\mathrm{K}$, et al. Diabetes mellitus and risk of stroke and its subtypes among Japanese: the Japan Public Health Centre study. Stroke 2011;42:2611-2614.

27. Shah AD, Langenberg C, Rapsomaniki E, et al. Type 2 diabetes and incidence of cardiovascular diseases: a cohort study in 1.9 million people. Lancet Diabetes Endocrinol 2015;3:105-114.

28. Rapsomaniki E, Timmis A, George J, et al. Blood pressure and incidence of twelve cardiovascular diseases: lifetime risks, healthy life-years lost, and age-specific associations in 1.25 million people. Lancet 2014;383:1899-1911.

29. Feigin V, Parag V, Lawes CMM, et al. Smoking and elevated blood pressure are the most important risk factors for subarachnoid hemorrhage in the Asia-Pacific region: an overview of 26 cohorts involving 306620 participants. Stroke 2005;36: $1360-1365$.

30. Tsai CF, Thomas B, Sudlow CLM. Epidemiology of stroke and its subtypes in Chinese vs white populations. Neurology 2013;81:264-272.

31. Thun MJ, Carter BD, Feskanich D, et al. 50-Year trends in smoking-related mortality in the United States. N Engl J Med 2013;368:351-364.

32. Wright FL, Green J, Canoy D, Cairns BJ, Balkwill A, Beral V. Vascular disease in women: comparison of diagnoses in hospital episode statistics and general practice records in England. BMC Med Res Methodol 2012:12:161. 


\title{
Differences in risk factors for 3 types of stroke
}

\author{
UK prospective study and meta-analyses
}

\begin{abstract}
Alison J. Price, DPhil, F. Lucy Wright, DPhil, Jane Green, DPhil, Angela Balkwill, MSc, Sau Wan Kan, MSc, TienYu Owen Yang, DPhil, Sarah Floud, PhD, Mary E. Kroll, DPhil, Rachel Simpson, MB, BCh,

Cathie L.M. Sudlow, FRCP(E), Valerie Beral, FRS, and Gillian K. Reeves, PhD
\end{abstract}

Correspondence

Dr. Price

alison.price@lshtm.ac.uk

Cite as: Neurology ${ }^{\circledR}$ 2018;90:e298-e306. doi:10.1212/WNL.0000000000004856

\section{Study question}

Do the effects of classic behavioral risk factors for vascular disease differ between subarachnoid hemorrhage, intracerebral hemorrhage, and ischemic stroke?

\section{Summary answer}

Diabetes mellitus increases the risk of intracerebral hemorrhage and ischemic stroke but decreases subarachnoid hemorrhage risk. Smoking increases the risk of all types of stroke, but more strongly for subarachnoid hemorrhage than the other types. Obesity increases ischemic stroke risk but decreases hemorrhagic stroke risk.

\section{What is known and what this paper adds}

Various behavioral factors can increase the overall risk of stroke, but it is unclear whether they have different effects on different types of stroke. In this study, substantial differences were found in the effects of diabetes mellitus, smoking, and adiposity on the risks of the 3 main types of stroke.

\section{Participants and setting}

This study followed up 712,433 women (mean age 59.8 [SD 4.9] years) with no history of stroke, heart disease, or cancer who participated in the prospective UK Million Women Study.

\section{Design, size, and duration}

The study analyzed data on lifestyle and other factors from questionnaires completed in 1999 to 2007, 3 years after recruitment. Women were followed up for a mean of 12.9 (SD 2.6) years. Outcome data were obtained by record linkage to nationwide health databases.

\section{Primary outcomes}

Primary outcomes were first recorded hospital admission or deaths attributed to subarachnoid hemorrhage, intracerebral hemorrhage, and ischemic stroke.

\section{Main results and the role of chance}

During follow-up, 8,128 women had an ischemic stroke, 2,032 had an intracerebral hemorrhage, and 1,536 had a subarachnoid hemorrhage. Diabetes mellitus increased the risk of ischemic stroke (relative risk [RR] 2.01, 95\% confidence interval [CI] 1.84-2.20) and intracerebral hemorrhage (RR 1.31, 95\% CI

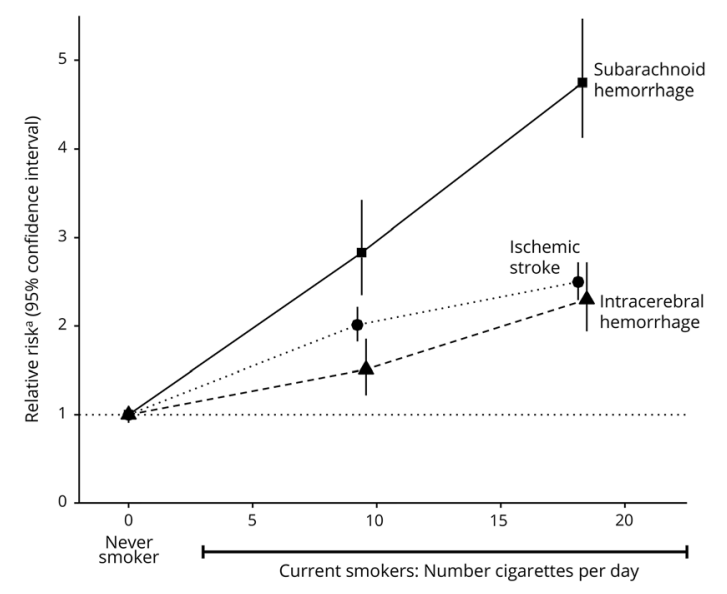

1.04-1.65) but decreased the risk of subarachnoid hemorrhage (RR 0.43, 95\% CI 0.26-0.69). Smoking $>15$ cigarettes per day increased the risk of subarachnoid hemorrhage (RR 4.75, 95\% CI 4.12-5.47) significantly more than for intracerebral hemorrhage (RR 2.30, 95\% CI 1.94-2.72) and ischemic stroke (RR $2.50,95 \%$ CI 2.29-2.72). Obesity was associated with an increased risk of ischemic stroke and a decreased risk of hemorrhagic stroke (heterogeneity $p<0.0001$ ).

\section{Bias, confounding, and other reasons for caution}

Analyses included strokes resulting in hospitalization or death, which is more likely for hemorrhagic than for ischemic strokes. However, this should not bias findings for specific stroke types.

\section{Generalizability to other populations}

Although our primary analyses were based on women, our meta-analyses showed similar findings for men and for European and Asian populations.

\section{Study funding/potential competing interests}

This study was funded by the UK Medical Research Council and Cancer Research UK. The authors report no competing interests. Go to Neurology.org/N for full disclosures. 


\section{Neurology}

\section{Differences in risk factors for 3 types of stroke: UK prospective study and meta-analyses}

Alison J. Price, F. Lucy Wright, Jane Green, et al.

Neurology 2018;90;e298-e306 Published Online before print January 10, 2018

DOI 10.1212/WNL.0000000000004856

\section{This information is current as of January 10, 2018}

\section{Updated Information \&} Services

References

Subspecialty Collections

Permissions \& Licensing

Reprints including high resolution figures, can be found at: http://n.neurology.org/content/90/4/e298.full

This article cites 29 articles, 11 of which you can access for free at: http://n.neurology.org/content/90/4/e298.full\#ref-list-1

This article, along with others on similar topics, appears in the following collection(s):

All global neurology

http://n.neurology.org/cgi/collection/all_global_neurology

Cohort studies

http://n.neurology.org/cgi/collection/cohort_studies

Infarction

http://n.neurology.org/cgi/collection/infarction

Intracerebral hemorrhage

http://n.neurology.org/cgi/collection/intracerebral_hemorrhage

Risk factors in epidemiology

http://n.neurology.org/cgi/collection/risk_factors_in_epidemiology Subarachnoid hemorrhage

http://n.neurology.org/cgi/collection/subarachnoid_hemorrhage

Information about reproducing this article in parts (figures,tables) or in its entirety can be found online at:

http://www.neurology.org/about/about_the_journal\#permissions

Information about ordering reprints can be found online:

http://n.neurology.org/subscribers/advertise

Neurology ${ }^{\circledR}$ is the official journal of the American Academy of Neurology. Published continuously since 1951 , it is now a weekly with 48 issues per year. Copyright Copyright (C) 2018 The Author(s). Published by Wolters Kluwer Health, Inc. on behalf of the American Academy of Neurology.. All rights reserved. Print ISSN: 0028-3878. Online ISSN: 1526-632X.

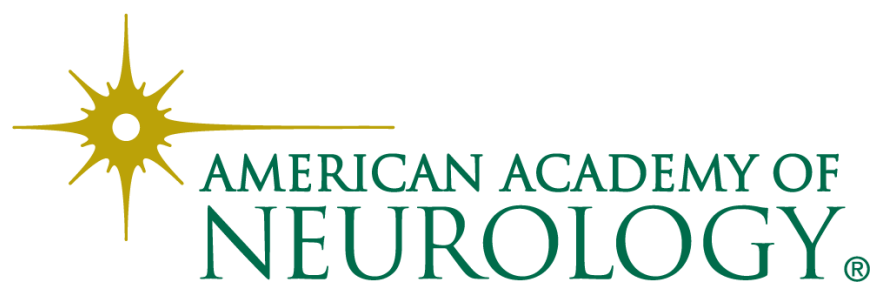

